\title{
Effects of Stereoisomers on Drug Activity
}

\author{
Rohan Kumar*, Maaz Hassan and Karan Pahuja \\ Department of Pharmacy, Benazir Bhutto Shaheed University Lyari, Karachi \\ *Corresponding author: Rohan Kumar, Department of Pharmacy, Benazir Bhutto Shaheed University Lyari, Karachi \\ To Cite This Article: Rohan Kumar, Maaz Hassan and Karan Pahuja. Effects of Stereoisomers on Drug Activity. Am J Biomed Sci \& Res. 2021 - \\ 13(3). AJBSR.MS.ID.001861. DOI: 10.34297/AJBSR.2021.13.001861.
}

Received: 眥 June 11, 2021; Published: 筒 June 21, 2021

\begin{abstract}
Chirality occupies an important position as it is a major factor in determining the role and synthesis of drugs. Chiral switch has helped in developing numerous drugs, it is amazing how two molecules that just differ in spatial arrangement of their atoms can have extremely different biological activities and thus extraordinary different effects on human body. In pharmaceutical industry many of the drugs are used as chiral products and many of them are marketed as racemates. Number of cardiovascular drugs like angiotensin converting enzyme inhibitors, calcium channel blockers and Beta-adrenergic blockers are used as chiral products. The chiral inversion is mainly of two types which include unidirectional and bidirectional. NSAIDs are the common example of unidirectional inversion while the drugs like temazepam, oxazepam and lorazepama re the examples of bidirectional inversion. Chiral molecules show stereo selective properties so these molecules differ in their metabolic pathways and mechanism of action. In the same way racemates work where one provides higher pharmacological actions than the other due to stereo selectivity. Choosing of enantiomers is a crucial picking as if chiral antidote chosen wrong may lead to carcinogenicity and teratogenicity [1,2].
\end{abstract}

\section{Introduction}

\section{Stereochemistry and Stereoisomers}

Molecules having atoms connected in same sequence but differing in arrangement of their atoms in space are the stereoisomers and consideration of such spatial aspects of molecules is stereochemistry or sub discipline of chemistry that is concerned with three dimensional arrangements of atoms and molecules and their effects in biological systems is the stereochemistry [3-6]. Cis and trans forms of alkenes are the major examples of stereoisomers. Stereoisomers occur either as enantiomers or as diastereomers. Former are the stereoisomers whose molecules are non-superposable mirror images of each other while the later ones do not have molecules which are mirror images of each other.

\section{Chirality and Enantiomers}

Two structurally similar forms of chemical molecules that behave very differently in biological systems are the enantiomers and geometric property of these molecules of not being superimposable with their mirror images is the chirality [7]. However, the molecules which overlay with their mirror image are said to be achiral. Very simply the right and left handed confirmation in most of the molecules is the chirality like DNA is a chiral molecule that turns in right handed way.
Chiral chemistry was introduced by Louis Pasteur when he separated two isomers of sodium ammonium tartrate in 1848 for the first time. Later it was known that chirality not only plays role in life of plants and animals but it also shows a profound role in pharmaceutical, agricultural and other chemical industries. Chirality matters very much in pharmaceutical sciences as most of the pharmaceuticals are chiral where if one mirror form provide desired therapeutic effect then the second one may be inactive or may provide lesser or even better therapeutic actions, in certain cases the second mirror form can show toxicity and adverse effects. In pharmaceutical science $56 \%$ of the drugs used are chiral and $88 \%$ of the last ones were marketed as racemic mixture of two equimolar enantiomers.

Simply chiral substances possess a unique architecture such that, despite sharing identical molecular formulas, atom to atom linkages and bonding distances they cannot be superimposed [8]. Thus, in the environment of living system where special structural activity relationships are required for effect (like: enzymes, receptors, transporters etc.) the physiochemical and biochemical properties of racemic mixtures and individual stereoisomer differ significantly. In the days now chemistry has been developed that much to differentiate between two enantiomers of drugs or 
between parent compounding and metabolites. Several drugs are now marketed as single enantiomer instead of previous racemic mixture, a process known as chiral switching $[9,10]$.

\section{Racemic Mixtures}

Around $25 \%$ of the marketed drugs are mixture of multiple agents rather than single chemical entity, these drugs are the mixture of more than 1 stereoisomer, these mixture forms of drugs are termed as racemic mixtures. Every single stereoisomer present in racemic form of drug differs in pharmacodynamics and pharmacokinetic properties with every other stereoisomer [11]. As racemic forms are there in order to provide better therapeutic actions, however; these may lead to increased adverse effects due to interactions between stereoisomers or any other reasons [1,2].

\section{Nomenclature of Chiral Compounds: the R, S-System}

Three chemists R. S. Cahn, C. K. Ingold and V. Prelog introduced $\mathrm{R}, \mathrm{S}$ system also called as Cahn-Ingold-Prelog rules of naming chiral compounds. $\mathrm{R}$ and $\mathrm{S}$ are from Latin words rectus and sinister meaning right and left respectively, both of these molecules have opposite configurations. The $\mathrm{R}, \mathrm{S}$ system is assigned in a way that all the atoms which are connected to chiral center are given priority according to atomic number. The higher the atomic number, the higher the priority. An arrow is drawn going from priority 1 to 2, 3 and so on, of the arrow moves clockwise then the absolute configuration is $\mathrm{R}$ and if the arrow goes anti-clockwise then the configuration is $\mathrm{S}$ [12-15].

\section{Chiral Drugs}

Chirality is one of the major factor for the synthesis and the role of the drugs and it may be possible that one of an enantiomer of a particular drug may be a medicine and on the other hand another enantiomer may be not only inactive but can cause toxic effect as well. In pharmaceutical industry many of the drugs which are currently used are chiral products and many of them are also marketed as the racemates consisting of an equimolar mixture of two enantiomers [16]. There are a number of cardiovascular drugs and agents which are used for the treatment of cardiac arrhythmias, hypertension, cardiac arrest and other cardiovascular diseases. Among these drugs the angiotensin-converting enzyme (ACE) inhibitors, Calcium channel blockers and $\beta$-adrenergic blocking agents are common [17]. Levorotatory isomer of all $\beta$-blockers is more potent in blocking $\beta$-adrenoreceptors than their dextrorotatory isomers such as S (-) propranolol is more active than its $\mathrm{R}(+)$ antipode. Many of the racemic forms of $\beta$ blockers are also used which includes betaxolol, metoprolol, atenolol etc. Many Calcium channel antagonists are used in racemic forms including felodipine, nicardipine and verapamil. There are mainly two kinds of chiral inversions in the drugs which includes unidirectional and bidirectional inversions. The most common example of unidirectional inversion is of NSAIDs including ketoprofen, ibuprofen and fenprofen etc. For this group only S-enantiomer is active and shows anti-inflammatory and analgesic properties. For example, S-ibuprofen is more potent as an inhibitor of COX 1 than R- ibuprofen. Bidirectional chiral inversion or racemization can be shown by temazepam, oxazepam and lorazepam. The antihypertensive drug like methyldopa has its effect in S isomer form and in case of penicillamine is acting as a highly potent agent for treating chronic arthritis and $\mathrm{R}$ isomer is highly toxic and has no therapeutic action as well. D-Ethambutol is an antituberclosis drug while L-Ethambutol can cause blindness. R-Thalidomide is sedative while S-Thalidomide can show teratogenic effects.

\section{Importance of Chiral Drugs in Biological Sys- tem}

Chirality has played a significant role in drug synthesis and production. The majority of the medicines that have been found are chiral. The association of drugs with biological targets such as proteins, nucleic acids, and bio membranes determines their pharmacological function [5].

One enantiomer of a chiral drug may be a treatment for a specific condition, whereas the other enantiomer may not only be ineffective but also poisonous. As a result, Chirality is crucial in drug development. In the design and synthesis of drugs, synthesizing a compound as a single enantiomer is important.

Drug History Therapeutic revolutions have taken place over the last 120 years. Drugs were used to heal and alleviate discomfort. With these, the misuse of certain medicines is increasing. The legislation has thus evolved to regulate the abuse of such medicines. Heroin was introduced for the first time in 1898. And Morphine was introduced in later years, aspirin, opium. Opium, morphine and cocaine contribute to overdose and mortality of many patent medicines. The calming syrup of Ms. Winslow kills several children because of overdose of morphine [6].

\section{Stereo selectivity in Chiral Molecules}

In drug deposition stereo selectivity has especially been observed for the processes which are interactive with the biological chiral macromolecules e.g. the active transportation mechanism, plasma protein binding and the metabolism of drugs. If, however, a chiral molecule is a substrate for an active transport mechanism, then a preferential absorption of the stereo isomer with a spatial arrangement like that of the natural substrate will be expected to cause differences in both isomer and radiometer [9].

In metabolism, stereo-differentiation is the law, and stereoselective metabolism is possibly the cause of the greater part of the disparity in enantioselective drug deposits, which results from a straightforward interaction between a drug and chiral 
macromolecules. The enantiomer of a drug administered as racemate will differ in pharmacokinetics profiles.

\section{Pharmacology of Chiral Drugs}

The body, with its many homochiral compounds, is an amazing chiral selector, because each racemic substance can interact with it differently, and each enantiomer will be metabolized by a different mechanism, resulting in different pharmacological activity. One isomer may therefore produce the desired therapy, while others may be inactive or cause unwanted or toxic effects in the worst cases [18].

Only racemic drugs will be investigated in the pharmacology field, and their operation will be divided into three classes. The majority of racemic pharmaceuticals have one large bioactive enantiomer (referred to as the eutomer), whereas the other is inactive or less active (referred to as the distomer), harmful, or can exert other desirable or undesired pharmacological properties. The second group is for medicines that are similarly active and have the same pharmacodynamics in both enantiomers. The final form is racemic drugs with only one eutomer, but the distomer can be converted into its bioactive antipode in the body through chiral inversion [19].

\section{Toxicology}

Enantiomeric selection to improve therapeutic effects or reduce drug toxicity has had mixed results in drug growth. Stereo selective metabolism of chiral compounds may also affect pharmacokinetics, pharmacodynamics, and toxicity, complicating genetic polymorphisms in drug disposition.

Racemization of optically pure pharmaceuticals may occur in vivo, negating single enantiomer benefits or causing unexpected side effects. For therapeutic advantage and to minimize adverse events, appropriate chiral antidotes must be chosen. Carcinogenicity and teratogenicity can vary between enantiomers [20].

Environmental toxicology provides several examples in which compound bioaccumulation, persistence, and toxicity show chiral dependence. In forensic toxicology, chiral analysis has been applied to illicit drug preparations and biological specimens, with the potential to assist in determination of cause of death and aid in the correct interpretation of substance abuse and "doping" screens.

\section{References}

1. Craig B, Scott AF A Handbook of Organic Chemistry by T.W. Graham Solomons Snyder

2. Nguyen LA, He H and Pham Huy C (2006) Chiral Drugs: An Overview from International Journal of Biomedical Science (IJBS). 2(2): 85-100.

3. McConathy J and Owens MJ (2003) Stereochemistry in drug action from the Primary Care Companion to the Journal of Clinical Psychiatry. 5(2): 70-73.

4. Hutt AJ (2002) The development of single isomer molecules: why and how. CNS Spectrums 7(S1): 14-22.

5. Bada JL (2015) Racemic Mixture from book Encyclopedia of Astrobiology. 2105-2105.

6. Simonyi M (1994) Stereo-chemical Definitions and Nomenclature: Changing signs? From Therapeutic Innovation and Regulatory Science 28(2): 533-540.

7. Nallamuthu A (2018) Role of Chirality in Drugs.

8. Jayakumar R, Vadivel R, Ananthi N (2018) Role of chirality in drugs: An overview from International Journal of Organic and Medicinal Chemistry.

9. Chhabra N, Aseri ML, Padmanabhan D (2013) A review of drug isomerism and its significance. Int J Appl Basic Med Res 3 (1): 16-18

10. Sharma B (2014) Nature of chiral drugs and their occurrence in environment. Journal of Xenobiotics. 4: 2272

11. Lin GQ, Zhang JG, Cheng JF (2011) Overview of chirality and chiral drugs from book Chiral drugs. Chemistry and Biological actions 3-28.

12. Leffingwell J (2003) Chirality and Bioactivity l. Pharmacology from project Chirality.

13. Tripathi KD (1993) Drug enantiomers: Configuration and pharmaceutical implications.

14. Smith SW (2009) Chiral Toxicology: it's the Same Thing...Only Different. Toxicol Sci 110(1): 4-30.

15. Howland RH (2009) Understanding Chirality and Stereochemistry Three-Dimensional Psychopharmacology. Journal of Psychological Nursing and Mental Health Services 47(7): 15-18.

16. Trucker GT (2000) Chiral switches. Lancet 355(9209): 1085-1087.

17. Patocka J, Ales D (2004) Biomedical aspects of chiral molecules. Journal of Applied Biomedicine 2(2).

18. Hardikar MS (2008) Chiral non-steroidal anti-inflammatory drugs - A review. J Indian Med Assoc 106(9): 615-624.

19. Liu Y, Gu XH (2011) Pharmacology of Chiral Drugs from book Chiral Drugs. Chemistry and Biological Action 323-345.

20. Yang G, Bu HZ (2011) Toxicology of Chiral Drugs from book Chiral Drugs. Chemistry and Biological Action 381-399. 